\title{
Derechos Humanos y Derecho Internacional
}

\author{
JUAN ANTONIO CARRILLO SALCEDO \\ Universidad de Sevilla
}

La aparición de un nuevo consenso internacional en torno a las nociones de derechos humanos y democracia, como valores generalmente aceptados por la comunidad de naciones en su conjunto, y la afirmación de que el ser humano es titular de derechos propios, oponibles jurídicamente a todos los Estados, constituyen extraordinarias innovaciones que hacen que, a diferencia del Derecho internacional clásico, la persona no pueda seguir siendo considerada como un mero objeto del Derecho internacional.

1.

La Carta de las Naciones Unidas contiene disposiciones que ponen de manifiesto innegables dimensiones de cambio y transformación en el Derecho internacional. Entre ellas se encuentra la proclamación de la dignidad de la persona y el respeto universal de los derechos humanos y de las libertades fundamentales como uno de los propósitos de la Organización (Preámbulo y artículos 1.3, $13.1,55$ c), 56, 62.2, 68, 73 y 76 de la Carta).

Tras la afirmación de estos propósitos y la posterior adopción de la Declaración Universal de Derechos Humanos por la Asamblea General de las Naciones Unidas el 10 de diciembre de 1948, la prevalencia de los Estados soberanos y la situación de dependencia de la persona respecto del Estado se han visto, simultáneamente, confirmadas y puestas en cuestión.

Confirmadas, ante todo, porque la estruetura interestatal sigue prevaleciendo en el Derecho internacional, y es a dicha estructura a la que la Carta de las Naciones Unidas vincula la noción de soberanía, presente en los principios de igualdad soberana de los Estados y no intervención en los asuntos que son esencialmente de la jurisdicción interna de los Estados (párrafos 1 y 7 del artículo 2 de la Carta).

Pero, al mismo tiempo, la prevalencia de los Estados soberanos y la precaria situdción jurídica de la persona humana ante el orden internacional se encuentran hoy puestas en cuestión ya que la afirmación de la dignidad de todo ser humano constituye una importante transformación del Derecho internacional en la medida en que, junto al clásico principio de la soberanía, ha aparecido otro principio constitucional del orden internacional contemporáneo: el de los derechos humanos. Éstos sobrepasan la limitación inherente al Derecho 
internacional clásico, que era ante todo un Derecho interestatal concebido por y para los Estados, esto es, el mínimo jurídico necesario para regular las relaciones de coexistencia y cooperación entre Estados soberanos territoriales. La innovación que en este orden de cosas introdujo la Carta de las Naciones Unidas -con la proclamación de la noción jurídica de la dignidad de todo ser humano y la afirmación del respeto universal de los derechos humanos, unida a la obligación de los Estados miembros de tomar medidas para la realización de dicho propósito- tenía necesariamente que incidir e influir en la naturaleza misma del Derecho internacional.

2.

El Derecho internacional tradicional, surgido en la Paz de Westfalia y consolidado entre 1815 y 1914, era un orden jurídico regulador de las relaciones de coexistencia y cooperación entre Estados soberanos, iguales e independientes, caracterizado por las siguientes rasgos:

1) las normas eran el producto del consentimiento, esto es, del acuerdo de voluntades de los Estados, manifestado de modo expreso en los tratados o de modo tácito en las costumbres;

2) dichas normas tenían por función distribuir y delimitar las competencias de los Estados, regulando sus derechos y deberes reciprocos;

3) las normas jurídicas internacionales, aunque obligatorias, eran de Derecho dispositivo por lo que los Estados podían modificarlas mediante acuerdos ya que no existía rango jerárquico alguno entre ellas;

4) cada Estado soberano, por último, apreciaba discrecionalmente el alcance de sus derechos y, en caso de violación de los mismos, podía recurrir unilateralmente a medidas de autoprotección o autotutela que podían implicar incluso el recurso a la guerra.

De este modo, los Estados, únicos sujetos del Derecho internacional, eran a la vez los creadores y los destinatarios de las normas jurídicas internacionales, pues la existencia de un legislador internacional resultaba impensable en un medio social integrado por Estados soberanos. A éstos quedaba confiada igualmente la aplicación del Derecho, en la medida en que el recurso al arbitraje era voluntario y la competencia del tribunal arbitral se basaba en el consentimiento de los Estados partes en una controversia. La ejecución del Derecho, por último, estaba asimismo en manos de los Estados que, en ejercicio de su derecho de autoprotección o autotutela, podían incluso recurrir libremente a la fuerza al no existir en el Derecho internacional de la época ni una prohibición del recurso a la guerra, ni una instancia internacional titular del monopolio del uso jurídico e institucionalizado de la coerción. 
De ahí el caracter fragmentario del Derecho internacional, que es consecuencia de la estructura paritaria y escasamente institucionalizada de la sociedad internacional. Las normas del Derecho internacional (en el que no existe un legislador, ni un juez, ni un mecanismo centralizado de aplicación de las reglas jurídicas) son, en efecto, relativas, en el triple sentido siguiente:

$\left.1^{\circ}\right)$ su alcance varía en función de las obligaciones asumidas por los Estados;

$2^{\circ}$ ) la apreciación de las situaciones jurídicas en que un Estado se encuentre implicado depende, en principio, de cada Estado, ya que las posiciones jurídicas y las pretensiones contradictorias de los Estados pueden coexistir dado que el sometimiento de una controversia al arbitraje o a la justicia internacional es voluntario y depende del consentimiento de los Estados;

$3^{\circ}$ ) finalmente, la sanción de los hechos ilícitos internacionales raramente toma la forma de una reacción social organizada e institucionalizada, ya que, en principio, cada Estado aprecia subjetivamente su posición jurídica frente a otro Estado y, cuando estima que un determinado ilícito internacional es atribuible a este último, puede adoptar, en las condiciones regladas por el Derecho internacional, las contramedidas que considere adecuadas.

En definitiva, estamos en presencia de una de las características esenciales del Derecho internacional: su relativismo. En principio, para que una obligación vincule a un Estado, o una situación produzca efectos jurídicos respecto de él, es preciso que dicho Estado haya participado en su creación o las haya reconocido; de ahí la relevancia del consentimiento en Derecho internacional, y que los Estados pretendan determinar cualquier punto controvertido, de hecho o de Derecho, según sus propios criterios. Estas pretensiones no prevalecen sobre la obligatoriedad de las normas del Derecho internacional, pero en cada situación aquéllas son valoradas por cada Estado, unilateral y subjetivamente, sin que las percepciones de un Estado prevalezcan automáticamente sobre las de otro.

Resulta así que, en sus comportamientos, los Estados tienen que respetar, y respetan normalmente, las normas jurídicas internacionales; pero a ellos corresponde examinar cuales son las exigencias del Derecho internacional en cada situación concreta. En el sistema jurídico internacional, por consiguiente, las situaciones y las obligaciones de los sujetos difieren según el Estado que se considere, ya que cada Estado aprecia unilateral y subjetivamente el alcance de sus obligaciones. Ello trae consigo que, en virtud del principio de equivalencia de las pretensiones, derivado de la igualdad soberana de los Estados, el orden jurídico internacional presente como una de sus características la de aparecer como una multiplicidad de representaciones subjetivas y divergentes; en consecuencia, el sistema internacional no es un conjunto coherente sino, más bien, 
un rompecabezas de alegaciones y pretensiones subjetivas que tienen, todas ellas, simplemente valor de presunciones ${ }^{1}$.

Obviamente, esta realidad, que se debe al carácter descentralizado y a la escasa institucionalización del Derecho internacional, no debe llevarnos a la errónea conclusión de que todas las posiciones jurídicas sostenidas por los Estados son igualmente válidas; pero sí debe hacernos conscientes de los rasgos de voluntarismo y discrecionalidad, de subjetivismo, que caracterizan a un ordenamiento jurídico, el Derecho internacional, en el que la soberanía de los Estados es un principio constitucional. De ahi la relevancia jurídica del consentimiento, de la voluntad de los Estados, el juego del principio de reciprocidad, y el relativismo del Derecho internacional: cada Estado pretende ejercer el conjunto de facultades y derechos que el Derecho internacional le reconoce y confiere, pero al hacerlo choca con las pretensiones de otros Estados; esta concurrencia muestra la razón de ser de la debilidad congénita al Derecho internacional, como consecuencia de su carácter descentralizado y escasamente institucionalizado.

En este orden de cosas, es indispensable que no pretendamos comprender y explicar el Derecho internacional en función del modelo de los ordenamientos jurídicos estatales, como si el Derecho interno fuese la única manifestación posible del fenómeno jurídico. Como todo ordenamiento jurídico, el Derecho internacional dispone de normas que regulan las relaciones entre sus sujetos, sus derechos y obligaciones, así como de principios, reglas y procedimientos relativos a cómo se elaboran y aplican las normas y cuáles son las reacciones que legítimamente son posibles en los supuestos de incumplimiento de las reglas jurídicas. Lo que ocurre es que, en principio y a diferencia de lo que pasa en los ordenamientos jurídicos de los Estados, estos procedimientos, principios y reglas no tienen carácter institucionalizado. Esto no significa, sin embargo, que el Derecho internacional no sea Derecho, ni tampoco que sea un Derecho primitivo, sino que es todavia, al menos en gran parte, el ordenamiento jurídico de un medio social descentralizado y escasamente institucionalizado.

3.

Ahora bien, desde fines de la Primera Guerra Mundial, y sobre todo a partir de 1945, el Derecho internacional ha venido experimentando un triple proceso de institucionalización, de socialización y de humanización que distancian profundamente al orden internacional contemporáneo de los rasgos que habían caracterizado al Derecho internacional tradicional. Un proceso de creciente institucionalización de la comunidad internacional, en primer lugar, como con-

\footnotetext{
${ }^{1}$ Weil, Prosper: "Le droit international en quête de son identité. Cours général de droit international public, en Recueil des Cours de l'Académie de Droit International, tomo 237 (1992-VI), pág. 55.
} 
secuencia del desarrollo y vigor de las Organizaciones Internacionales, universales y regionales. En segundo lugar, un proceso de socialización del Derecho internacional en la medida en que éste regula hoy relaciones sociales y humanas más complejas y amplias que las tradicionales relaciones políticas entre Estados soberanos. Por último, un proceso de humanización del orden internacional debido a que el Derecho internacional ha comenzado a dar entrada a los pueblos y a la persona, rompiendo así el exclusivismo tradicional de los Estados como únicos sujetos del Derecho internacional. El desarrollo del fenómeno de Organización Internacional, la progresiva ampliación de las materias reguladas por el Derecho internacional, y la creciente relevancia de la persona han incidido y modificado la naturaleza, estructura y funciones del Derecho internacional que en muchos de sus principios inspiradores es hoy muy diferente del Derecho internacional clásico.

Por otra parte, estos procesos de cambios en el Derecho internacional no actúan aisladamente sino en interacción, influyéndose recíprocamente. En consecuencia, el Derecho internacional no puede hoy ser comprendido exclusivamente como un sistema jurídico regulador de las relaciones entre Estados porque la vida y las relaciones internacionales se han hecho más complejas 2 .

Desde la perspectiva de estas últimas, en efecto, han tenido lugar cambios que diferencian profundamente al mundo contemporáneo del que terminó prevaleciendo en el siglo XVII, cuando surgió históricamente lo que hoy llamamos Derecho internacional, así como del existente entre el Congreso de Viena y el comienzo de la Primera Guerra Mundial, lapso en el que se consolidó lo que denominamos Derecho internacional tradicional. Entre los cambios a que acabo de hacer referencia, destacan los siguientes:

1. Los Estados parecen demasiados pequeños respecto de los grandes problemas, y demasiado grandes con relación a los de la vida cotidiana, con lo que asistimos a un debilitamiento de la centralidad del Estado en las relaciones internacionales.

2. Los Estados, además, se ven puestos en cuestión al comprobarse que son incapaces de resolver por sí mismos problemas globales que exigen un esfuerzo de cooperación internacional, y al verificarse que existen otras entidades no estatales (empresas transnacionales; Organizaciones Internacionales No Gubernamentales; etc.) que operan con creciente relevancia en la vida internacional.

3. Heterogeneidad del sistema internacional, frente a la relativa homogeneidad que le había caracterizado a lo largo de la historia, cuando estaba constituido casi exclusivamente por Estados soberanos territoriales.

${ }^{2}$ Carrillo Salcedo, J.A. "Permanence et mutations en droit international", en Boutros Boutros-Ghali Amicorum Discipulorumque Liber. Paix, Développment, Démocratie, Bruylant, Bruselas 1998, Vol. I, págs. 291-304 
4. Creciente importancia en las relaciones internacionales de los factores económicos, científico- técnicos y culturales.

5. Proceso de difusión del poder, que implica cambios importantes tanto en la naturaleza del poder como en la distribución del mismo entre los distintos actores de la vida internacional, que hoy no son exclusivamente los Estados.

6. Progresiva difuminación, e incluso desaparición, de los límites entre el mundo interno de los Estados y el mundo internacional, esto es, entre la política interior y la política exterior.

7. Revalorización de lo humano y de lo humanitario como valores generalmente compartidos y como dimensión de las relaciones internacionales.

8. Aparición de un nuevo consenso internacional en torno a las nociones de derechos humanos y democracia, como valores generalmente aceptados por la comunidad internacional en su conjunto.

En esta compleja realidad, los Estados se presentan con rasgos muy distintos de los que les venían caracterizando desde la Paz de Westfalia, en 1648. En primer lugar, los Estados que pretendieron entonces la plenitud de soberanía e independencia, se encuentran hoy subordinados en razón de los límites que les impone la realidad de la interdependencia; en segundo lugar, tras haber proclamado su integridad territorial tienen que admitir que sus fronteras han perdido mucho de su carácter cerrado, pues los hechos les obligan a tomar conciencia de que se hallan sumergidos en la transnacionalización de la vida; por último, después de haber afirmado durante siglos su unidad política, que sólo excepcionalmente quedaba perturbada, muchos sufren las consecuencias de la dislocación de tal unidad ${ }^{\beta}$.

\section{4 .}

La revalorización de lo humano y de lo humanitario como dimensión de las relaciones internacionales, la aparición de un consenso en torno a las nociones de derechos humanos y democracia como valores generalmente aceptados por la comunidad internacional en su conjunto, y la afirmación de que el ser humano es titular de derechos propios, oponibles jurídicamente a todos los Estados, incluso al Estado del que sea nacional o al Estado bajo cuya jurisdicción se encuentre, constituyen extraordinarias innovaciones que hacen que, a diferencia del Derecho internacional clásico, la persona no pueda ser considerada como un mero objeto del Derecho internacional.

\footnotetext{
${ }^{3}$ Dupuy, René-Jean: "Le Conseil de Sécurité en recherche de la paix", en Les Nations Unies et la restauration de l'Etat, Paris 1995, págs. 7 y ss.
} 
No se trata de afirmar que el individuo sea hoy sujeto pleno del Derecho internacional. Existe, sin duda, una cierta subjetividad internacional de la persona, pero cuando esto ocurre, estamos todavía ante supuestos excepcionales y ante una subjetividad limitada y funcional. No comparto, por consiguiente, las doctrinas que sostienen la plena subjetividad internacional del individuo, y pienso en este orden de cosas que aunque la vía de progreso consiste en ir generalizando y reforzando los regímenes convencionales en los que la persona que se considere víctima de una violación de sus derechos internacionalmente reconocidos pueda demandar ante una instancia internacional al Estado al que dicha violación sea atribuible, incluso si es el Estado del que la persona en cuestión sea nacional (como ocurre en el sistema de garantía instituido en el Convenio Europeo de Derechos Humanos), lo esencial de una concepción personalista del Derecho internacional no está en este problema técnico (que depende de la legitimación del individuo ante instancias jurisdiccionales internacionales de garantía y control), sino en afirmar, el lugar supremo del interés humano en el orden de los valores. Lo importante estriba, en mi opinión, en la obligación de los Estados de hacer de dicho interés humano un principio de cooperación más concreto, más directamente accesible que la idea general de justicia, pero no menos imperativo por su significación civilizadora y alcance universal, y en la progresiva cristalización en el Derecho internacional contemporáneo de la idea de que, en materia de derechos humanos, los Estados tienen obligaciones hacia la comunidad internacional en su conjunto.

Creo que éste es el significado jurídico de la afirmación hecha por la Corte Internacional de Justicia en su sentencia de 5 de febrero de 1970, al admitir la existencia de obligaciones de los Estados hacia la comunidad internacional en su conjunto, esto es, de obligaciones erga omnes que resultan, por ejemplo, de la prohibición de los actos de agresión y del genocidio, así como de los principios y reglas relativos a los derechos fundamentales de la persona humana4.

La jurisprudencia de la Corte Internacional de Justicia ha hecho reiteradas referencias a estos principios generales en los que ha basado su afirmación de que existen en Derecho internacional obligaciones que vinculan a los Estados al margen de su consentimiento, o incluso en contra de su voluntad. Así, en su sentencia de 9 de abril de 1949, relativa al asunto del Estrecho de Corfú (Reino Unido de Gran Bretaña c. Albania), la Corte se había referido a ciertos " principios generales bien reconocidos, tales como elementales consideraciones de humanidad, más absolutos en tiempos de paz que en tiempos de guerra" ${ }^{5}$.

En el mismo sentido, la Corte sostuvo en su Opinión consultiva de 18 de mayo de 1951, relativa a la Validez de ciertas reservas a la Convención para la prevención y sanción del delito de genocidio, que dicha Convención expresa

${ }^{4}$ C.I.J., Recueil 1970, parágrafos 33 y 34 de la sentencia relativa al asunto de la Barcelona Traction, Bélgica contra España.

${ }^{5}$ C.I.J., Recueil 1949, p. 29. 
y contiene "principios reconocidos por las naciones civilizadas, obligatorios para todos los Estados incluso al margen de todo vínculo convencional" ${ }^{6}$. Más tarde, en la sentencia de 27 de junio de 1986 relativa al asunto de las actividades militares y paramilitares en y contra Nicaragua (Nicaragua c. Estados Unidos de América) - una sentencia que con acierto ha sido calificada de faro del Derecho internacional contemporáneo- la Corte sostuvo una concepción no voluntarista del Derecho internacional cuando afirmó que los Estados Unidos de América estaban obligados a respetar, e incluso a hacer respetar, las Convenciones de Ginebra de 1949, de Derecho internacional humanitario, pues tal obligación "no resulta sólo de los Convenios en sí, sino también de los principios generales del Derecho humanitario de los que los Convenios en cuestión no son más que la expresión concreta" 7 .

Por último, la Corte ha sostenido en la Opinión consultiva de 8 de julio de 1996 sobre Licitud de la amenaza o el empleo de armas nucleares (solicitada por la Asamblea General de las Naciones Unidas), que los principios cardinales que constituyen la esencia del Derecho humanitario se expresan tanto en normas convencionales que se han transformado en normas consuetudinarias como en principios del Derecho internacional. De ahí que la Corte concluyera afirmando que todos los Estados "han de cumplir esas normas fundamentales, hayan o no ratificado los convenios que las estatuyen, porque constituyen principios intransgredibles del derecho internacional consuetudinario" ${ }^{\text {. }}$.

La resuelta posición de la Corte Internacional de Justicia, órgano judicial principal de las Naciones Unidas, permitió al Institut de Droit International sostener, en el artículo $1^{\circ}$ de su Resolución sobre La protección de los derechos humanos y el principio de no intervención en los asuntos internos de los Estados (adoptada en la sesión de Santiago de Compostela, el 13 de septiembre de 1989), que "Los derechos humanos son la expresión directa de la dignidad de la persona humana. La obligación de los Estados de asegurar su respeto se desprende del propio reconocimiento de esta dignidad proclamada en la Carta de las Naciones Unidas y en la Declaración Universal de Derechos Humanos. Esta obligación internacional es, según la fórmula utilizada por la Corte Internacional de Justicia, una obligación erga omnes: incumbe a todo Estado con respecto a la comunidad internacional en su conjunto, y todo Estado tiene un interés jurídico en la protección de los derechos humanos" ${ }^{9}$.

i

${ }^{6}$ C.IJ., Recueil 1951, pp. 23-24.

7 I.C.J., Reports 1986, parágrafo 220 de la sentencia.

${ }^{8}$ Parágrafo 79 de la Opinión consultiva. La cursiva es mía.

${ }^{9}$ Annuaire de l'Institut de Droit International, Vol. 63-II, Sesión de Santiago de Compostela, 1989, págs. 338-344. 
5.

Estas afirmaciones de la Corte Internacional de Justicia son del mayor interés porque confirman la irreductible necesidad de una respuesta distinta de la voluntarista al problema del fundamento del Derecho internacional ya que, por su misma naturaleza, la norma jurídica implica siempre una parte de heteronomía y, por consiguiente, de objetivismo.

Creo por ello que no es posible ignorar la honda significación de los principios generales del Derecho internacional a la hora de explicar la razón de ser de la obligatoriedad del Derecho internacional. Sobre la base de la jurisprudencia de la Corte Internacional de Justicia, pienso que es posible distinguir tres tipos de principios generales: en primer lugar, los que trasladan al Derecho internacional conceptos o reglas heredados de los ordenamientos internos; en segundo lugar, los principios que la jurisprudencia utiliza como formulación lapidaria de una costumbre; y en tercer lugar, por último, los principios que son la afirmación jurisprudencial de la necesidad de una norma. En este último supuesto, el de los principios "fundamentales" o "esenciales" del Derecho internacional (que obviamente son algunos de los principios generales, pero no todos), se está en presencia de una concepción objetivista del Derecho internacional general.

Ésta es la razón por la que, en mi Curso general en la Academia de Derecho Internacional de La Haya, en 1996, sostuve que la Corte no ignora desde luego el papel del consentimiento de los Estados en la elaboración del Derecho internacional, pero que, progresivamente, "elle s'est forgé une conception des principes généraux du droit selon laquelle ces derniers ne sont pas les seuls à être reconnus dans les ordres juridiques internes; le sont aussi ceux qui existent et font l'objet d'une application en tant que principes du droit international, dont le caractère obligatoire ne dépend pas du consentement des Etats" 10 .

Este núcleo de principios generales del Derecho internacional, expresión de una conciencia jurídica común, se forma al margen tanto de la práctica generalmente aceptada como del acuerdo de los Estados. Pero no por ello dejan de ser Derecho vigente, ya que los principios generales del Derecho internacional ponen de manifiesto que, por su misma naturaleza, esta parte esencial del Derecho internacional, la más importante de este ordenamiento jurídico, se impone desde el exterior de la voluntad de los Estados e implica siempre una irreductible dimensión de heteronomía y, por consiguiente, de objetivismo. Reconozco que, como ha observado el profesor Antonio Enrique Pérez Luño, la expresión principios generales del Derecho tiene siempre carác-

\footnotetext{
${ }^{10}$ Carrillo Salcedo, J.A.: "Droit international et souveraineté des Etats. Cours général de droit international public, en Recueil des Cours de l'Académie de Droit International, tomo 257 , pág. 156.
} 
ter ambiguo y contradictorio y que es inherente a ella una pluralidad significativa; pero como él, pienso que asistimos hoy a una indiscutible revalorización de los principios generales y que, en su dimensión axiológica, tales principios pueden ser entendidos como los prima principia, axiomas o postulados éticos que deben inpirar todo el orden jurídico, bien porque el Derecho se remite a ellos como término o canon de aspiración o porque forman parte integrante del ordenamiento ${ }^{11}$. En mi opinión, los principios generales del Derecho internacional son en definitiva la expresión de una conciencia jurídica común, y constituyen una manifestación más - junto con la aceptación de normas imperativas o de ius cogens en Derecho internacional contemporáneo- del proceso al que con tanto acierto se refirió el profesor Truyol y Serra en su Curso general en la Academia de Derecho internacional de La Haya: el eterno retorno del Derecho natura ${ }^{2}$. Una concepción iusnaturalista del Derecho en la que, tras las vicisitudes del pasado - en el que no ha faltado la hybris de un criticable triunfalismo- se ha transformado en un "droit assagi et irréductible", y cuyos principios se ven hoy, en un tiempo histórico de cambio social, como un factor de progreso.

\section{6.}

Pero si los derechos humanos son una de las dimensiones constitucionales del Derecho internacional contemporáneo, con el alcance jurídico que acabo de señalar basándome en la jurisprudencia de la Corte Internacional de Justicia, ¿qué cambios ha introducido este nuevo principio en la posición de los Estados ante el Derecho internacional y en un sistema jurídico que había sido concebido para regular las relaciones de coexistencia y de cooperación entre los Estados y cuya estructura de base, a pesar de los cambios experimentados y las transformaciones introducidas por las Organizaciones Internacionales, continúa siendo fundamentalmente interestatal? ${ }^{13}$.

Creo que la soberanía de los Estados sigue siendo un principio constitucional del Derecho internacional, y que no ha sido desplazada de esta posición por el reconocimiento de la dignidad de la personna, simbolo de las transformaciones del orden internacional que habitualmente denominamos proceso de huma-

${ }^{11}$ Pérez Luño, A.: "Los principios generales del Derecho: ¿Un mito jurídico?", en Revista de Estudios Políticos, 1997, págs. 9-24.

${ }^{12}$ Truyol Serra, A.: "Théorie du droit international public. Cours général", en Recueil des Cours de l'Académie de Droit International, tomo 183 (1981-IV), págs. 142-143.

${ }^{13}$ En mi monografia Soberania de los Estados y derechos humanos en Derecho internacional contemporáneo (Editorial Tecnos, Madrid 1995) me esforcé en responder a estas preguntas analizando la tensión existente entre soberanía de los Estados y derechos humanos en torno a cinco sectores del Derecho internacional: fuentes, reglas imperativas y obligaciones erga omnes, aplicación de las normas, responsabilidad internacional de los Estados y, finalmente, la aplicación coercitiva del Derecho, esto es, las reacciones de los Estados y de la comunidad internacional organizada respecto de las violaciones graves de derechos humanos. 
nización del Derecho internacional. Pero pienso que sí ha quedado erosionada y relativizada por el desarrollo normativo que ha seguido a las disposiciones de la Carta de las Naciones Unidas en materia de derechos humanos, ya que en Derecho internacional general contemporáneo (y por tanto, no sólo en el marco convencional), todos los Estados tienen la obligación jurídica, omnium y erga omnes, de respetar los derechos fundamentales de toda persona que se encuentre bajo su jurisdicción.

Por consiguiente, si el trato que un Estado diera a sus nacionales era en el Derecho internacional tradicional una cuestión de jurisdicción interna (ya que el Derecho internacional no regía esta cuestión y se limitaba a regular la posición jurídica de los extranjeros), en el Derecho internacional contemporáneo ocurre lo contrario como consecuencia del desarrollo progresivo que el orden internacional ha experimentado en materia de reconocimiento y protección internacional de los derechos humanos. De ahí que el Institut de Droit International pudiera sostener, en el párrafo primero del artículo segundo de la Resolución antes citada, que ningún Estado que viole la obligación de Derecho internacional general de respetar los derechos humanos fundamentales de toda persona que se encuentre bajo su jurisdicción, cualquiera sea su nacionalidad e incluso si carece de ella, podrá sustraerse a su responsabilidad internacional con el pretexto de que esta materia es esencialmente de su jurisdicción intern $a^{14}$. En definitiva, la evolución experimentada por el Derecho internacional tras la proclamación de la dignidad de la persona en la Carta de las Naciones Unidas ha traido consigo un retroceso espectacular de la competencia exclusiva de los Estados y, por tanto, de su soberanía, en la medida en que el reconocimiento internacional de los derechos humanos ha sometido a los estados a obligaciones internacionales que condicionan el ejercicio de su soberania territorial, a pesar del carácter exclusivo de esta última ${ }^{15}$.

Todo ello implica un cambio en la posición constitucional de la soberanía estatal ante el orden internacional, que hoy comienza a registrar jurídicamente el deber de solidaridad entre todos los Estados a fin de garantizar la protección universal y efectiva de los derechos humanos, en los términos del artículo 56 de la Carta de las Naciones Unidas. En el innegable desarrollo normativo experimentado por el Derecho internacional de los derechos humanos, lo esencial estriba en recordar dos datos jurídicos: en primer lugar que, como dijera la Corte Permanente de Justicia Internacional en su Opinión consultiva de 7 de febrero de 1923 relativa a los decretos de nacionalidad en Túnez y Marruecos, "la cuestión de saber si una determinada materia entra o no en el ámbito de competencia exclusiva de un Estado es una cuestión relativa que depende del desarrollo del Derecho internacional ${ }^{16}$; en segundo lugar que, como dijera

\footnotetext{
${ }^{14}$ Annuaire de I'Institut de Droit International, Vol. 63-II, Sesión de Santiago de Compostela, 1989, págs. 338-344.

${ }_{15}$ Dupuy, Pierre-Marie: Droit International Public, 4a ed., Dalloz, Paris 1998 , págs. 161-162.

${ }^{16}$ C.P.J.I., Série $B, \mathrm{n}^{\circ} 4$, págs. $24-25$.
} 
el árbitro Max Huber en la sentencia arbitral de 4 de abril de 1928 relativa al asunto Isla de Palmas, la soberanía implica derechos y deberes ${ }^{17}$.

Los deberes y obligaciones de los Estados soberanos están en función del desarrollo del Derecho internacional y de ahí que, en mi opinión, la tensión dialéctica entre soberanía de los Estados, de una parte, y los derechos humanos, de otra, se resuelva hoy de la siguiente forma: por ser soberanos, los Estados tienen obligaciones internacionales en materia de derechos humanos respecto de la comunidad internacional en su conjunto. La dificultad estriba en que si la integración de los derechos humanos en el Derecho internacional ha ampliado el ámbito de este último, dicha integración ha politizado al mismo tiempo el problema, y sería equivocado ver en ella únicamente un triunfo de la ética sobre la política; lo inverso es igualmente cierto, pues la protección de los derechos humanos se ha convertido también en política de los derechos humanos y ésta, como toda política, es selectiva y discriminatoria ${ }^{18}$.

Influencia de factores políticos, subjetivos, especialmente relevante en un orden jurídico escasamente institucionalizado como es el Derecho internacional. De aquí la debilidad intrínseca a nociones innovadoras como las de reglas de ius cogens, obligaciones erga omnes, crímenes internacionales; etc.; de aquí también, la prudencia - en mi opinión excesiva y criticable- con que la Corte Internacional de Justicia ha abordado hasta ahora el problema de las reglas imperativas de Derecho internacional, como la sentencia de 30 de junio de 1995 en la controversia entre Portugal y Australia o la opinión consultiva de 8 de julio de 1996, sobre la Licitud de la amenaza o el empleo de armas nucleares, han puesto de manifiesto ${ }^{19}$.

En todo caso, y a pesar de estas limitaciones y de las debilidades congénitas del Derecho internacional - que es un sistema jurídico cuyos caracteres estructurales se corresponden con las exigencias de un medio social predominantemente interestatal-, me parece innegable que los derechos humanos han supuesto la ampliación del ámbito de materias regidas por el Derecho internacional, ampliación que encierra dentro de sí una modificación de la naturaleza de dicho ordenamiento jurídico. El Derecho internacional sigue siendo un sistema jurídico interestatal, pero las relaciones entre el poder público y los particulares que se encuentren bajo su jurisdicción son de ahora en adelante una materia regida por normas jurídicas internacionales. Este hecho va más allá de una simple ampliación del ámbito de materias reguladas por el Derecho internacional, ya que incide en su naturaleza, que ha dejado de ser exclusivamente interestatal, y en el fundamento de su carácter obligatorio que ya no puede ser reducido exclusivamente a la voluntad de los Estados.

: Ello supone una especie de reencuentro con los clásicos del Derecho de Gentes, y en especial con el pensamiento de Francisco de Vitoria, en la medida

\footnotetext{
${ }^{17}$ Reports of International Arbitral Awards, Vol. II, pág. 839.

${ }^{18}$ Carrillo Salcedo, J.A.: loc. cit. en nota 10, pág. 47.

${ }_{19}$ Carrillo Salcedo, J.A.: loc. cit. en nota 10, págs. 135- 140.
} 
en que la referencia a la persona y a la comunidad internacional tiende a sustituir el concepto clásico de sociedad internacional (como grupo social atomizado y fragmentado, compuesto por un tejido de relaciones bilaterales dominadas por los intereses nacionales, la reciprocidad, y el do ut des), por la visión de una comunidad interdependiente, unida y solidaria, que comienza a ser una realidad histórica, o al menos un orden en potencia, y deja de ser un mito o una mera aspiración en el espíritu de los hombres. 